\title{
Resíduos agrícolas incorporados a substrato comercial na produção de mudas de repolho
}

\author{
Agricultural residues incorporated to commercial substrates in the production of cabbage seedlings
}

\section{Lorena Aparecida Merlo Meneghelli ${ }^{*}$, Paola Alfonsa Vieira Lo Monaco ${ }^{2}$, Marcelo Rodrigo Krause ${ }^{1}$, Caroline Merlo Meneghelli ${ }^{1}$, Louise Pinto Guisolfi ${ }^{1} \&$ Juliana Menegassi ${ }^{2}$}

${ }^{1}$ Universidade Federal de Viçosa, Viçosa, MG, Brasil. *Autor para correspondência: Iorena.merlo@hotmail.com.

${ }^{2}$ Instituto Federal de Educação, Ciência e Tecnologia do Espírito Santo, Santa Teresa, ES, Brasil.

\begin{abstract}
RESUMO
Na produção de hortaliças de qualidade a formação de mudas é uma das fases mais importantes para o ciclo da cultura, influenciando diretamente no desempenho final da planta, tanto do ponto de vista nutricional como produtivo. Um dos fatores decisivos para obtenção de mudas com qualidade e consequente aumento de produtividade é o tipo de substrato utilizado. $O$ estudo objetivou avaliar o efeito de proporções crescentes de resíduos da secagem de grãos de café (moinha) em substratos alternativos compostos de fibra de coco, casca de ovo e substrato comercial, nas variáveis de crescimento de mudas de repolho. O delineamento experimental foi o inteiramente casualizado, com cinco tratamentos e dez repetições, sendo os tratamentos: T0: substrato comercial (testemunha); T1: 10\% de moinha (MO) + 15\% fibra de coco (FC) $+5 \%$ casca de ovo (CO) $+70 \%$ substrato comercial (SC); T2: $20 \%$ de $\mathrm{MO}+15 \% \mathrm{FC}+$ $5 \%$ CO + 60\% SC; T3: $30 \%$ de MO + 15\% FC + 5\% CO + 50\% SC; T4: $40 \%$ de MO + $15 \%$ FC + $5 \%$ CO + $40 \%$ SC. As variáveis avaliadas foram condutividade elétrica do substrato, diâmetro de coleto, número de folhas, altura de plantas e matérias secas da parte aérea, raiz e total de planta. Os maiores valores obtidos das variáveis analisadas foram obtidos no tratamento 4 , no qual se utilizou-se $40 \%$ de moinha + $15 \%$ fibra de coco $+5 \%$ casca de ovo $+40 \%$ substrato comercial.
\end{abstract}

PALAVRAS-CHAVE: Brassica oleracea, moinha, fibra de coco, casca de ovo.

\begin{abstract}
In the production of quality vegetables, the formation of seedlings is one of the most important phases for the crop cycle, directly influencing the final performance of the plant, both from a nutritional and productive point of view. One of the decisive factors to obtain seedlings with quality and consequent increase in productivity is the type of substrate used. The objective of this research was to evaluate the effect of increasing proportions of residues from the drying of coffee beans (chaff) on alternative substrates constituted by coconut fiber, eggshell and commercial substrate, in the growth variables of cabbage seedlings. The experiment was conducted under a completely randomized design, with five treatments and ten replicates, with the following treatments: T0: commercial substrate (control); T1: $10 \%$ chaff $(\mathrm{CH})+15 \%$ coconut fiber (CF) $+5 \%$ eggshell (ES) $+70 \%$ commercial substrate (CS); T2: $20 \% \mathrm{CH}+15 \% \mathrm{CF}+5 \% \mathrm{ES}$ $+60 \%$ CS; T3: $30 \%$ CH + $15 \%$ CF + $5 \%$ ES + 50\% CS; T4: $40 \%$ CH + $15 \%$ CF + $5 \%$ ES + $40 \%$ CS. The evaluated variables were substrate electrical conductivity, collection diameter, number of leaves, height of plants and dry matter of shoot, root, and total plant. The highest values obtained from the variables analyzed were obtained in the treatment 4 , in which $40 \%$ of woodwool $+15 \%$ coconut fiber $+5 \%$ eggshell $+40 \%$ commercial substrate were used.
\end{abstract}

KEYWORDS: Brassica oleracea, chaff, coconut fiber, eggshell.

\section{INTRODUÇÃO}

O repolho (Brassica oleracea var. capitata) está entre as principais fontes de alimento consumidas nos países desenvolvidos, sendo cultivado tanto por meio da agricultura familiar, quanto pelos grandes produtores de hortaliças.

Na produção de hortaliças de qualidade, a formação de mudas é uma das fases mais importantes 
para o ciclo da cultura, influenciando diretamente no desempenho final da planta, tanto do ponto de vista nutricional como produtivo (CAMPANHARO et al. 2006).

Um dos fatores decisivos para obtenção de mudas com qualidade e consequente aumento de produtividade é o tipo de substrato utilizado. De acordo com MEDEIROS et al. (2010) o substrato deve proporcionar eficiência na germinação e emergência de plântulas, além de fornecer suprimento adequado de nutrientes, oxigênio e eliminação do $\mathrm{CO}_{2}$. Para que isto seja obtido, o substrato deve ser provido de boas características físicas, químicas, biológicas e sanitárias para obtenção de mudas de qualidade (SANTOS et al. 2010, LOPES et al. 2012, MESQUITA et al. 2012).

Na produção de mudas de repolho, os substratos comerciais são amplamente utilizados, no entanto, constituem-se em um custo a mais na etapa de produção de mudas. Como a maioria do cultivo das hortaliças no Brasil é realizado por agricultores familiares, em pequena escala, os custos com substratos levam a uma diminuição na rentabilidade do investimento, sugerindo a necessidade de se buscar novas fontes alternativas de substratos.

Nesse sentido, muitos estudos vêm sendo realizados visando o aproveitamento de resíduos agrícolas na composição de substratos para a produção de mudas de repolho, tais como nos trabalhos de SOARES et al. (2009) e COSTA et al. (2014). Além de reduzir custos, é uma forma de minimizar o impacto ambiental causado pelo descarte de forma inadequada desses resíduos no ambiente, tornando a propriedade agrícola sustentável.

Dentre os resíduos gerados em elevada quantidade no Espírito Santo, destaca-se a moinha de café. De acordo com MENEGHELLI et al. (2016), a moinha é composta por restos vegetais tais como folhas, galhos, restos de inflorescências e grãos mal formados do próprio cafeeiro, que, quando secados juntamente com o mesmo, sofrem queima e são liberados do secador. Os mesmos autores, ao caracterizarem quimicamente esse material, constataram elevadas concentrações de potássio, fósforo e especialmente nitrogênio. Em razão da disponibilidade e do potencial fertilizante da moinha, formula-se a hipótese que este resíduo pode ser um componente promissor na substituição de parte do substrato comercial, numa proporção que carece de experimentação.

Além da moinha de café, resíduos como a casca de ovo e a fibra de coco também são gerados em elevada quantidade no Estado do Espírito Santo, e possuem atributos favoráveis na composição do substrato, juntamente com a moinha de café e o substrato comercial. Segundo MEDEIROS \& ALVES (2014) a casca de ovo é composta por substâncias orgânicas e inorgânicas. Dentre as inorgânicas, destaca-se o carbonato de cálcio, podendo ser utilizado como um dos componentes nutricionais para formulação de um substrato. Quanto à fibra de coco, MACHADO et al. (2014) salientam o caráter renovável, a elevada biodegradabilidade, as propriedades mecânicas melhoradas e o baixo custo deste resíduo.

Como é praticamente impossível encontrar um substrato com todas as características desejáveis (ARAÚJO NETO et al. 2009), acredita-se que a mistura desses resíduos, com diferentes atributos, pode ser benéfica. Nesse caso, há a necessidade de se verificar experimentalmente, para cada espécie vegetal, qual o substrato ou a melhor mistura de substratos que permita obter mudas de qualidade, tal como sugerido por ARAÚJO NETO et al. (2009).

Assim, objetivou-se, com a execução deste trabalho, avaliar o efeito de proporções crescentes de resíduos da secagem de grãos de café (moinha) em substratos alternativos compostos de fibra de coco, casca de ovo e substrato comercial, nas variáveis de crescimento de mudas de repolho.

\section{MATERIAL E MÉTODOS}

O experimento foi implantado e conduzido no viveiro de produção de mudas do Instituto Federal do Espírito Santo, Campus Santa Teresa, no município de Santa Teresa, Espírito Santo (1848' S; 4040' O; $130 \mathrm{~m}$ de altitude). O clima, segundo a classificação de Köppen, enquadra-se no tipo Cwa (subtropical de inverno seco), com temperatura média anual de $24,6{ }^{\circ} \mathrm{C}$ e precipitação média anual variando entre 700 a $1200 \mathrm{~mm}$. A temperatura e a umidade relativa do ar no período experimental variaram de 19,9 a $38,2{ }^{\circ} \mathrm{C}$ e $47,5 \%$ a $69,5 \%$, respectivamente. Na Tabela 1 apresenta-se a análise química do substrato comercial.

Os resíduos utilizados no substrato alternativo para produção de mudas de repolho foram provenientes da secagem de grãos de café, denominado "moinha", a fibra de coco e a casca de ovo. A moinha foi peneirada utilizando uma peneira de metal de granulométrica de $3 \mathrm{~mm}$ em média. A fibra de coco foi seca ao ar e processada em desintegrador. A casca de ovo foi triturada em almofariz, até se obter um farelo na forma de pó.

A caracterização química e físico-química do substrato comerical e dos resíduos utilizados na composição dos substratos foi realizada no Laboratório de Solos e Resíduos Sólidos do Departamento de 
Engenharia Agrícola da Universidade Federal de Viçosa. A análise físico-química consistiu na determinação da condutividade elétrica (CE), por meio de um condutivímetro de bancada. A análise química consistiu na determinação do $\mathrm{pH}$, por meio de um potenciômetro de bancada, e na quantificação das concentrações de carbono orgânico facilmente oxidável $\left(\mathrm{CO}_{\mathrm{fo}}\right)$, carbono orgânico total $\left(\mathrm{CO}_{T}\right)$, nitrogênio total $\left(\mathrm{N}_{\mathrm{T}}\right)$, fósforo $(\mathrm{P})$ e potássio (K), seguindo-se metodologia descrita por MATOS (2015). Na Tabela 1 apresentam-se os atributos químicos e físico-químico da moinha, da fibra de coco e da casca de ovo utilizadas no experimento.

Tabela 1. Atributos químicos e físico-químico de moinha (MO), fibra de coco (FC), casca de ovo (CO) e substrato comercial (SC) utilizadas no experimento.

Table 1. Chemical and physico-chemical attributes of chaff (CH), coconut fiber (CF), egg shell (ES) and commercial substrate were used in the experiment.

\begin{tabular}{|c|c|c|c|c|c|c|c|c|c|}
\hline \multirow{2}{*}{ Resíduos } & \multirow{2}{*}{$\mathrm{pH}$} & CE & $\mathrm{CO}_{\mathrm{Fo}}$ & $\mathrm{CO}_{\mathrm{T}}$ & MO & $\mathrm{N}_{T}$ & $P$ & K & $\mathrm{Ca}+\mathrm{Mg}$ \\
\hline & & $\mathrm{dS} \mathrm{m}^{-1}$ & & \multicolumn{6}{|c|}{ 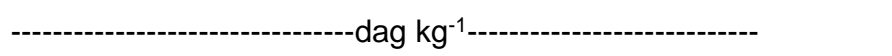 } \\
\hline MO & 5,60 & 6,49 & 45,3 & 58,9 & 101,5 & 3,70 & 0,140 & 0,71 & - \\
\hline $\mathrm{FC}$ & 7,15 & 0,09 & 57,1 & 74,1 & 127,7 & 0,66 & 0,053 & 0,14 & - \\
\hline $\mathrm{CO}$ & 9,37 & 0,37 & 2,8 & 3,6 & 6,2 & 0,87 & 0,084 & 0,06 & 31,9 \\
\hline SC & 5,62 & - & - & 21 & 52,21 & 0,62 & 1,55 & 0,44 & - \\
\hline
\end{tabular}

$\overline{\mathrm{pH}}=$ potencial hidrogeniônico em água; $\mathrm{CE}=$ condutividade elétrica; $\mathrm{COfo}=$ carbono orgânico facilmente oxidável; $\mathrm{CO}_{\mathrm{T}}=\mathrm{carbono}$ orgânico total; $\mathrm{MO}$ = matéria orgânica; $\mathrm{N}_{\mathrm{T}}=$ nitrogênio total; $\mathrm{P}$ = fósforo; $\mathrm{K}$ = potássio; $\mathrm{Ca}+\mathrm{Mg}$ = cálcio + magnésio.

O delineamento experimental escolhido foi o inteiramente casualizado, com cinco tratamentos e dez repetições. Cada unidade experimental consistiu de 20 mudas, totalizando 1000 mudas em todo o experimento. Foram consideradas úteis seis plantas centrais para cada unidade experimental. Os tratamentos foram T0: Substrato comercial (testemunha); T1: $10 \%$ de moinha $+15 \%$ fibra de coco $+5 \%$ casca de ovo $+70 \%$ substrato comercial; T2: $20 \%$ de moinha $+15 \%$ fibra de coco $+5 \%$ casca de ovo + $60 \%$ substrato comercial; T3: $30 \%$ de moinha $+15 \%$ fibra de coco $+5 \%$ casca de ovo $+50 \%$ substrato comercial; T4: $40 \%$ de moinha $+15 \%$ fibra de coco $+5 \%$ casca de ovo $+40 \%$ substrato comercial. A quantidade de resíduos adicionada aos tratamentos foi calculada com base no volume, utilizando, como recipiente de medida um béquer.

Como a moinha é um resíduo com elevada disponibilidade e de baixo custo na região Centro Serrana do Espírito Santo, estudou-se proporções crescentes deste resíduo no sentido de diminuir a quantidade de substrato comercial no composto. As proporções estudadas (não superiores a $40 \%$ ) justificam-se em razão da elevada condutividade elétrica deste resíduo (Tabela 2). Como a quantidade de resíduos adicionada aos tratamentos foi baseada no volume, optou-se por uma proporção fixa de casca de ovo de apenas 5\%, já que proporções maiores poderiam prejudicar a aeração do substrato. Já a proporção fixa de $15 \%$ de fibra de coco foi escolhida para completar o volume de $100 \%$ no substrato.

As sementes de repolho utilizadas foram da variedade "Repolho 60 dias", sendo semeadas em bandejas de poliestireno expandido (isopor) com 200 células, colocando-se duas sementes por célula. Utilizou-se o sistema de produção de mudas em bandejas suspensas, alocadas em bancadas de alvenaria, irrigadas manualmente duas vezes ao dia, pela manhã e à tarde. Não foi realizada qualquer adubação com fertilizantes. O viveiro onde foi desenvolvido o trabalho era telado com $50 \%$ de sombreamento.

Aos 16 dias após a semeadura (DAS) realizou-se um desbaste deixando-se a plântula mais vigorosa em cada célula. As avaliações ocorreram aos 33 DAS consistindo-se na determinação do diâmetro de coleto (DC), condutividade elétrica (CE) do substrato, na contagem do número de folhas (NF), na medição da altura de plantas (AP) e nas quantificações das matérias secas da parte aérea (MSPA), raiz (MSR) e total (MST) das plantas.

O DC foi obtido por meio de um paquímetro digital de precisão. A CE dos substratos foi determinada por meio de um condutivímetro, na proporção de resíduo e água de 1:2,5, seguindo-se metodologia descrita por MATOS (2015). Para a obtenção da altura de plantas foi utilizada uma régua milimetrada, medindo-se da base do colo até a gema apical que deu origem à última folha. A MSPA foi obtida através do corte das mudas próximas ao substrato. Para a obtenção da MSR, as raízes foram cuidadosamente lavadas em água corrente. Posteriormente, os materiais foram acondicionados em sacos de papel e alocados em estufa com 
circulação de ar forçado a $65{ }^{\circ} \mathrm{C}$ por 72 horas. Em seguida, foi realizada a pesagem dos materiais em balança eletrônica de precisão de 0,0001 g.

Todas as variáveis avaliadas foram submetidas aos testes de normalidade (Lilliefors) e de homocedasticidade (Barttlet), estes, pressupostos para a validação da análise de variância. Aquelas que não atenderam aos requisitos foram transformadas em raiz quadrada, sendo novamente submetidas aos mesmos testes de validação. Para as variáveis que novamente não atenderam aos pressupostos, optou-se pela realização do teste não paramétrico de Kruskal Wallis, contemplando-se a avaliação dos efeitos dos tratamentos por postos.

Dada a diferença qualitativa entre o tratamento de substrato comercial (T0) e os demais (T1 a T4), as comparações entre o grupo destes últimos e o T0 (Testemunha) foram estabelecidas por meio do contraste entre estes tratamentos (T0 contra os demais) obtido da decomposição das somas de quadrados dos tratamentos, na análise de variância. Além destas, para o caso de efeitos significativos $(p<0,05)$ para as comparações entre os tratamentos T1, T2, T3 e T4, relacionados ao nível de moinha, adotaram-se seus ajustes em modelos de regressão pelo método dos polinômios ortogonais. Para todos os procedimentos foi adotado um " $\alpha$ " igual a 0,05. Todas as análises estatísticas foram realizadas por meio programa SISVAR 5.6. (FERREIRA 2015) e ASSISTAT 7.7 (SILVA 2016).

\section{RESULTADOS E DISCUSSÃO}

De acordo com os resultados apresentados na Tabela 2, observa-se que o grupo de tratamentos onde a moinha esteve presente foi superior àquele com uso exclusivo do substrato comercial, para todas as variáveis avaliadas, já que os estimadores dos contrastes para cada variável foi negativo e significativo $(p<0,05)$. Tais resultados indicam que os substratos contendo proporções crescentes de moinha incorporadas ao substrato comercial, juntamente com a fibra de coco e casca de ovo, podem substituir o uso exclusivo do substrato comercial, devido suas características físicas e químicas.

Tabela 2. Coeficientes, níveis de significância e estimadores dos contrastes entre o tratamento de substrato comercial, e aqueles com diferentes níveis de inserção da moinha no substrato.

Table 2. Coefficients, levels of significance and estimators of the contrasts between commercial substrate treatment, and those with different levels of chaff insertion in the substrate.

\begin{tabular}{|c|c|c|c|c|c|c|c|}
\hline & \multicolumn{5}{|c|}{ Tratamentos } & \multirow[b]{3}{*}{ Estimador } & \multirow[b]{3}{*}{ Erro a } \\
\hline & \multicolumn{5}{|c|}{ Níveis de Moinha } & & \\
\hline & Conv. & $10 \%$ & $20 \%$ & $30 \%$ & $40 \%$ & & \\
\hline \multirow[t]{2}{*}{ Coeficientes do Contraste } & 4 & -1 & -1 & -1 & -1 & - & - \\
\hline & \multicolumn{5}{|c|}{ Diâmetro do coleto (mm) } & & \\
\hline \multirow[t]{2}{*}{ Médias dos tratamentos } & 0,42 & 0,69 & 0,88 & 0,97 & 1,14 & $-1,58$ & 0,000 \\
\hline & \multicolumn{5}{|c|}{ Matéria seca de parte aérea (mg) } & & \\
\hline \multirow[t]{2}{*}{ Médias dos tratamentos } & 5,19 & 6,56 & 7,40 & 7,95 & 8,50 & $-4,44$ & 0,000 \\
\hline & \multicolumn{5}{|c|}{ Matéria seca total de planta (mg) } & & \\
\hline \multirow[t]{2}{*}{ Médias dos tratamentos } & 6,85 & 8,37 & 9,34 & 10,29 & 10,31 & $-4,08$ & 0,000 \\
\hline & \multicolumn{5}{|c|}{ Condutividade elétrica $\left(\mathrm{mS} / \mathrm{cm}^{-1}\right)$} & & \\
\hline Médias dos tratamentos & 4,08 & 5,89 & 7,79 & 9,32 & 10,81 & $-13,42$ & 0,000 \\
\hline
\end{tabular}

Na Figura 1 apresenta-se o diâmetro de coleto (DC), massa seca de parte aérea (MSPA), massa seca total de planta (MSTP) e condutividade elétrica do substrato (CE), respectivamente. As variáveis ligadas à matéria seca de parte aérea, matéria seca total de planta e condutividade elétrica do substrato, por não atenderem aos pressupostos da análise de variância, foram transformadas em suas raízes quadradas e apresentadas nas análises de regressão.

De acordo com a análise de regressão, foram observados efeitos de primeiro grau $(p<0,05)$ para todas as variáveis analisadas em mudas de repolho. De um modo geral, verifica-se que o incremento de 
moinha nos substratos alternativos proporcionou valores crescentes no DC (Figura 1A) e na MSPA (Figura 1B) e MST (Figura 1B), de mudas de repolho.

$\mathrm{Na}$ fase de muda, o DC é um parâmetro muito importante para avaliar a rusticidade da espécie (ROSA et al. 2009), além de ser uma característica observada para indicar a capacidade de sobrevivência das mudas no campo (D'AVILA et al. 2011). Nesse sentido, verifica-se que o T4 proporcionou o maior valor de diâmetro do coleto $(1,135 \mathrm{~mm})$. A moinha possui elevado potencial de aproveitamento na agricultura, contendo elevadas concentrações de nutrientes sobre tudo $\circ \mathrm{N}$, o que pode ter contribuído para que 0 tratamento 4, contendo $40 \%$ de moinha, obtivesse o maior valor de diâmetro de coleto.

Resultados semelhantes foram encontrados por SOARES et al. (2009), que ao avaliarem substratos alternativos a base de composto orgânico e pó de rocha na produção de mudas de repolho, obtiveram o melhor desempenho no tratamento com $100 \%$ de composto, com valores de DC variando de 0,91 a 1,13 $\mathrm{mm}$, aos $46 \mathrm{DAS}$. Esta característica é importante, pois as folhas constituem uma das principais fontes de fotoassimilados e nutrientes para a sobrevivência e adaptação da muda. De acordo com a Figura 1B, o tratamento 4 proporcionou o maior valor médio estimado de MSPA (8,56 mg, equivalendo a 73,27 mg na escala original), dentre os tratamentos aplicados.

A quantidade de matéria seca é bastante variável entre espécies e na avaliação da qualidade de mudas, é um dos parâmetros que define a habilidade de sobrevivência após o plantio. A MST da muda é resultado da capacidade fotossintética e da quantidade de nutrientes minerais absorvidos (LOPES et al. 2013). De acordo com a Figura. O tratamento 4 proporcionou o maior valor estimado de MST $(10,59 \mathrm{mg}$ equivalente $122,2 \mathrm{mg}$ na escala original), dentre os tratamentos aplicados.
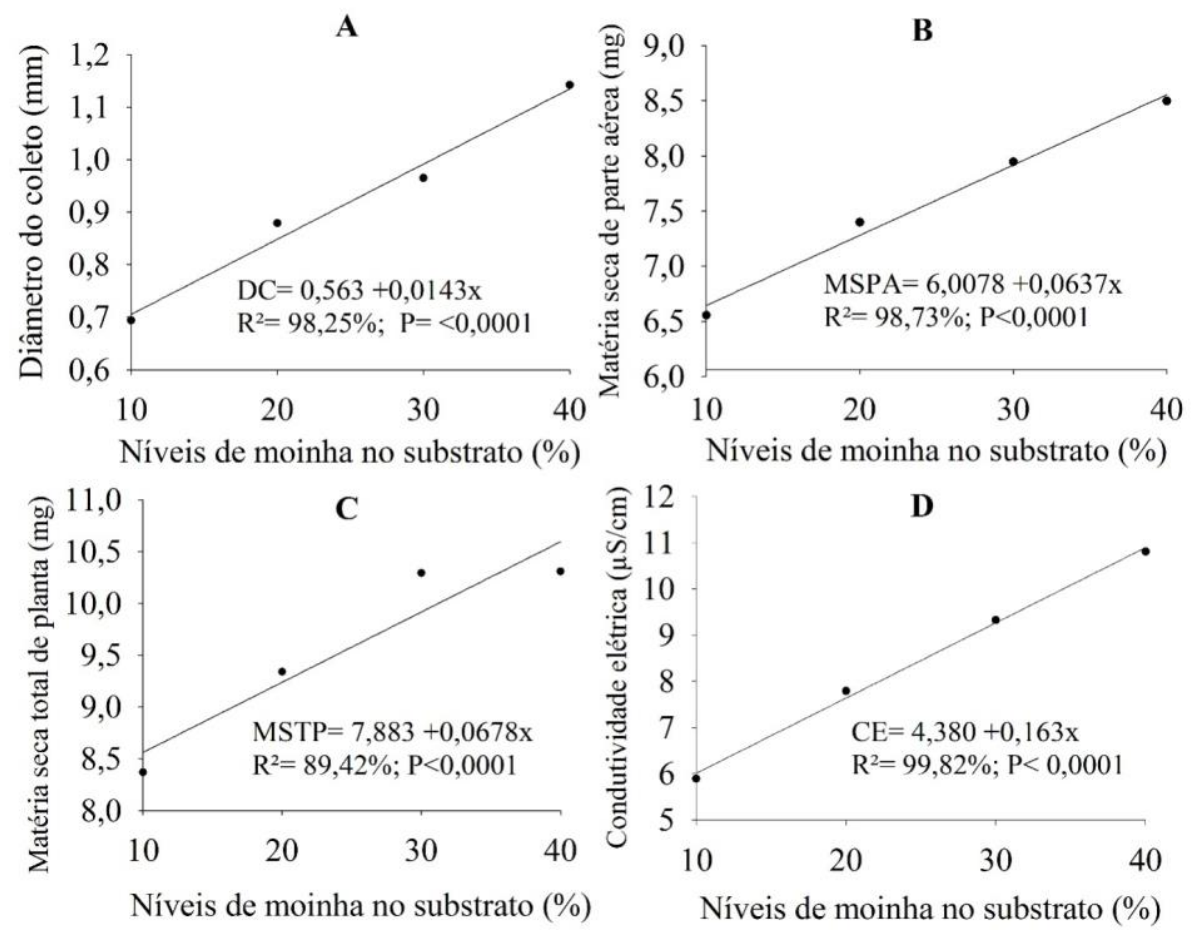

Figura 1. Diâmetro de coleto (A), matéria seca de parte aérea (B), matéria seca total $(C)$ e condutividade elétrica (D) em função das porcentagens crescentes de moinha incorporadas ao substrato contendo casca de ovo, fibra de coco e substrato comercial.

Figure 1. Stem diameter (A), dry matter of aerial part (B), total dry matter (C) and electrical conductivity (D) as a function of increasing percentages of chaff incorporated in the substrate containing eggshell, coconut fiber and commercial substrate.

Tal como observado para as outras variáveis, o incremento de moinha no substrato proporcionou valores crescentes de condutividade elétrica, sendo o T4, o tratamento que proporcionou maior valor estimado de CE $\left(10,9 \mu \mathrm{Scm}^{-1}\right.$ estimado e $188,8 \mu \mathrm{S} \mathrm{cm} \mathrm{cm}^{-1}$ na escala original). Na Tabela 2, verifica-se que apesar dos efeitos positivos da utilização da moinha no substrato comercial, nas variáveis de crescimento das mudas, houve um aumento da condutividade elétrica do substrato. $O$ aumento da condutividade elétrica do substrato pode interferir negativamente no crescimento da maioria das plantas de interesse comercial. $O$ excesso de sais na zona radicular, prejudica a germinação, desenvolvimento e produtividade das plantas. Isso porque uma maior concentração da solução exige da planta um maior dispêndio de energia para 
conseguir absorver água (efeito osmótico) prejudicando seus processos metabólicos essenciais. Mesmo assim, quando a moinha esta misturada a outros resíduos ela não alcança valores que possam causar prejuízos ao desenvolvimento das mudas de repolho.

Verifica-se, através da Figura 2, que para a variável NF os tratamentos contendo concentrações crescentes de moinha ( $T 1, T 2, T 3, T 4)$ foram estatisticamente superiores ao tratamento testemunha (T0) e não diferiram entre si $(p<0,05)$. Para a variável AP (Figura 2B), os tratamentos T4, T3 e T2 demonstraramse superiores aos demais, respectivamente, e estes não diferiram entre si $(p<0,05)$.

Os resíduos utilizados na composição do substrato devem manter uma interação com a planta (OLIVEIRA et al. 2013) e essa interação entre substrato-planta quem faz são as raízes. Para que isso ocorra de forma eficaz, o substrato deve propiciar o desenvolvimento do sistema radicular ao longo de toda a cédula, tornando o enraizamento uniforme. Assim, a plântula explorará um maior volume de substrato e poderá absorver mais nutrientes. De acordo com COSTA et al. (2013), as raízes com adequado desenvolvimento suportam melhor as mudanças de ambiente, pois o sistema radicular das plantas permite maior área de contato com o solo, justificado pela maior MSR. De acordo com a Figura 2 o T3 foi superior aos T0 e T1 e não diferiu dos tratamentos contendo T4 e T2 $(p<0,05)$.

De um modo geral, verifica-se que os maiores valores obtidos nas variáveis analisadas foi no T4. Tal fato pode estar associado à elevada diversidade de nutrientes existentes na moinha, notadamente o $\mathrm{N}$ (Tabela 1). $O$ incremento da área foliar das plantas de milho com o aumento das concentrações de nitrogênio ocorrem, pois este nutriente desempenha papel importante na nutrição, contribuindo no processo de crescimento e desenvolvimento das plantas (FRANÇA et al. 2011). ALEMAN \& CHAVES (2016) reforçam que aumentos na MSPA são esperados em função da aplicação de doses de N, principalmente por esse nutriente contribuir para o crescimento vegetativo, expansão foliar e taxa de crescimento do caule. Além disso, resíduos como a casca de ovo e a fibra de coco são importantes componentes na qualidade física do substrato, proporcionando melhor desenvolvimento das mudas.

A

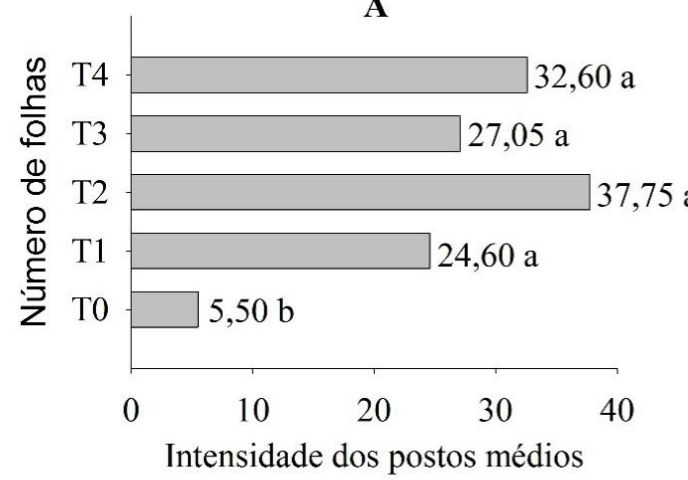

C

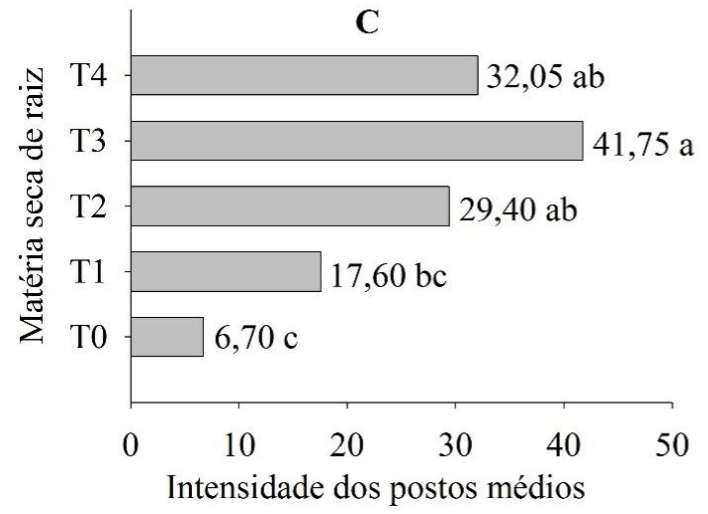

B

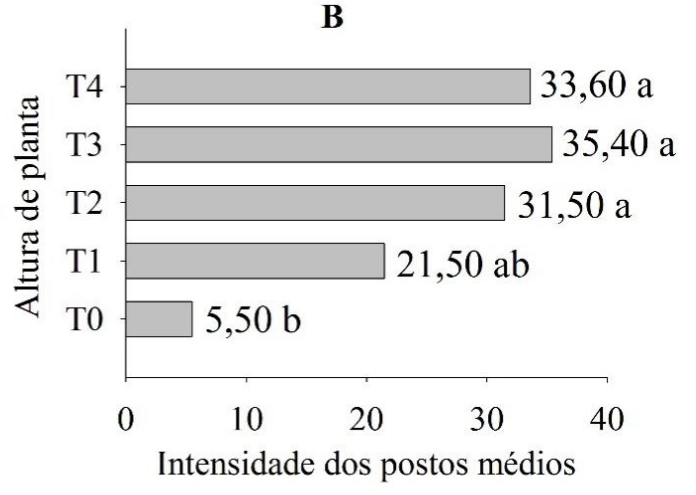

Figura 2. Comparações dos postos médios para as variáveis: (A) número de folhas, (B) altura de planta, (C) matéria seca de raiz por meio do teste não-paramétrico de Kruskal Wallis a $5 \%$ de probabilidade. Os postos médios seguidos de mesmas letras não diferem entre si, pelo teste SNK a $5 \%$ de probabilidade.

Figure 2. Comparisons of the average positions for the variables: (A) number of leaves, (B) plant height, (C) root dry matter by Kruskal Wallis non-parametric test at 5\% probability. The average positions followed by the same letters do not differ from each other, according to the SNK test at 5\% probability. 
Além da moinha, a incorporação da casca de ovo e da fibra de coco possam ter contribuído positivamente para desenvolvimento das mudas. A casca de ovo, embora tenha menor conteúdo de matéria orgânica entre os resíduos utilizados, contribui com nutrientes, especialmente com ○ Ca (Tabela 1), fundamental na síntese das paredes celulares, na lamela média e divisão celular, além de exercer funções em vários processos celulares, como no controle da transcrição e na liberação de sinais químicos (TAIZ \& ZEIGER 2013). Já a fibra de coco, pode ter contribuído não somente com a qualidade química do substrato (Tabela 1), como também com a qualidade física do substrato, proporcionando adequada aeração e retenção de água. COUTO et al. (2012) reforçam que este resíduo tem demonstrado elevado potencial no cultivo vegetal em razão da elevada porosidade e retenção de umidade.

\section{CONCLUSÃO}

O substrato alternativo contendo $40 \%$ de moinha $+15 \%$ fibra de coco $+5 \%$ casca de ovo $+40 \%$ substrato comercial, proporcionou os maiores valores para todas as variáveis avaliadas.

\section{REFERÊNCIAS}

ALEMAN CC \& CHAVES TC. 2016. Efeito da adubação nitrogenada via fertirrigação em capim limão. Nucleus 13: 199204.

ARAÚJO NETO SE et al. 2009. Produção de muda orgânica de pimentão com diferentes substratos. Ciência Rural 39: 1408-1413.

CAMPANHARO $\mathrm{M}$ et al. 2006. Características físicas de diferentes substratos para produção de mudas de tomateiro. Caatinga 19: 140-145.

COSTA LAM et al. 2013. Avaliação de substratos para a produção de mudas de tomate e pepino. Revista Ceres 60 : 675-682.

COSTA LAM et al. 2014. Substratos alternativos para produção de repolho e beterraba em consórcio e monocultivo. Revista Brasileira de Engenharia Agrícola e Ambiental 18: 150-156.

COUTO TR et al. 2012. Resíduos da agroindústria como substrato na aclimatização de mudas micropropagadas de bromélia. Revista Brasileira de Ciências Agrárias 7: 242-246.

D'AVILA FS et al. 2011. Efeito do potássio na fase de rustificação de mudas clonais de eucalipto. Revista Árvore 35: 1319.

FERREIRA DF. 2015. Sisvar. Versão 5.6. Lavras: UFLA/DEX.

FRANÇA S et al. 2011. Nitrogênio disponível ao milho: Crescimento, absorção e rendimento de grãos. Revista Brasileira de Engenharia Agrícola e Ambiental 11: 1143-1151.

LOPES JC et al. 2012. Broccoli production depending on the seed production system and organic and mineral fertilizer. Horticultura Brasileira 30: 143-150.

LOPES EC et al. 2013. Growth of mangrove seedlings under different levels of shading at the Peninsula of Ajuruteua, Bragança, Pará. Acta Amazonica 43: 291-296.

MACHADO BAS et al. 2014. Obtenção de nanocelulose da fibra de coco verde e incorporação em filmes biodegradáveis de amido plastificados com glicerol. Química Nova 37: 1275-1282.

MATOS AT. 2015. Manual de análise de resíduos sólidos e águas residuárias. 1.ed. Viçosa: Editora UFV. 149p.

MEDEIROS AS et al. 2010. Utilização de compostos orgânicos para uso como substratos na produção de mudas de alface. Revista Agrarian 3: 261-266.

MEDEIROS FM \& ALVES MGM. 2014. Qualidade de ovos comerciais. Revista Eletrônica Nutri Time 11: 3515-3524.

MENEGHELLI CM et al. 2016. Resíduo da secagem dos grãos de café como substrato alternativo em mudas de café conilon. Coffee Science 11: 330-335.

MESQUITA EF et al. 2012. Produção de mudas de mamoeiro em função de substratos contendo esterco bovino e volumes de recipientes. Revista Brasileira de Ciências Agrárias 7: 58-65.

OLIVEIRA JR et al. 2013. Húmus de minhoca associado a composto orgânico para a produção de mudas de tomate. Revista Agrogeoambiental 5: 79-86.

ROSA LS et al. 2009. Emergência, crescimento e padrão de qualidade de mudas de Schizolobium amazonicum Huber ex Ducke sob diferentes níveis de sombreamento e profundidades de semeadura. Revista de Ciências Agrárias 52: 87-98.

SANTOS MR et al. 2010. Produção de mudas de pimentão em substratos à base de vermicomposto. Bioscience Journal 26: $572-578$.

SILVA FAS. 2016. Assistat 7.7. Campina Grande. UFCG.

SOARES LR et al. 2009. Avaliação de Substratos Alternativos para Produção de Mudas de Repolho. Revista Brasileira de Agroecologia 4: 1780-1783.

TAIZ L \& ZEIGER E. 2013. Fisiologia vegetal. 5.ed. Porto Alegre: Artmed. 954p. 